\title{
Neighborhood Isolation during the COVID-19 Pandemic
}

\author{
Thomas Marlow, ${ }^{a}$ Kinga Makovi, ${ }^{a}$ Bruno Abrahao ${ }^{b, c}$
}

a) New York University Abu Dhabi; b) New York University Shanghai; c) New York University

\begin{abstract}
The COVID-19 pandemic has disrupted Americans' daily mobility, which could contribute to greater social stratification. Relying on SafeGraph cell phone movement data from 2019 and 2020, we use two indices proposed by Phillips and colleagues (2019) to measure mobility inequality between census tracts in the 25 largest U.S. cities. These measures capture the importance of hubs and neighborhood isolation in a network. In the earliest phases of the pandemic, neighborhood isolation rapidly increased, and the importance of downtown central business districts declined. Mobility hubs generally regained their importance, whereas neighborhood isolation remained elevated and increased again during the latter half of 2020. Linear regression models with city and week fixed effects find that new COVID-19 cases are positively associated with neighborhood isolation changes a week later. Additionally, places with larger populations, more public transportation use, and greater racial and ethnic segregation had larger increases in neighborhood isolation during 2020.
\end{abstract}

Keywords: mobility inequality; urban sociology; networks; COVID-19

Citation: Marlow, Thomas, Kinga Makovi, and Bruno Abrahao. 2021. "Neighborhood Isolation during the COVID-19 Pandemic." Sociological Science 8: 170-190.

Received: March 14, 2021

Accepted: April 22, 2021

Published: June 14, 2021

Editor(s): Jesper Sørensen, Mario Small

DOI: $10.15195 / \mathrm{v} 8 . \mathrm{a9}$

Copyright: (C) 2021 The Author(s). This open-access article has been published under a Creative Commons Attribution License, which allows unrestricted use, distribution and reproduction, in any form, as long as the original author and source have been credited. (C) (i)
THE COVID-19 pandemic is a tremendously disruptive period for the everyday 1 lives of most Americans. Many of the disruptions to daily life have direct implications for how people move in cities (Gao et al. 2020). Changes in mobility patterns are an important potential mechanism linking the pandemic to observed increases in other forms of social inequality (Chetty et al. 2020). Although prior work has already documented changes in mobility patterns during the pandemic (Badr et al. 2020; Chang et al. 2021; Gao et al. 2020; Glaeser, Gorback, and Redding 2020; Gupta et al. 2020; Nguyen et al. 2020), this article provides the first rigorous empirical exploration of changes in mobility patterns using cell phone data to describe how the COVID-19 pandemic altered inequalities in mobility networks in the 25 largest cities in the United States during 2020. Furthermore, we show how these changes correlate with disease progression and other factors that describe cities.

With the growth of large data sets that allow researchers to track the movement of millions of individuals, a growing body of sociological research focuses on developing theory and methods to understand the forms and consequences of everyday mobility (Browning, Pinchak, and Calder 2021; Cagney et al. 2020; Levy, Phillips, and Sampson 2020; Prestby et al. 2020; Phillips et al. 2019; Wang et al. 2018). In this line of work mobility refers to the aggregate daily patterns of travel between neighborhoods. Inequality in a city's mobility network then is conceptualized as any deviation from random movement between neighborhoods. Inequality in mobility has been described along two dimensions. First, deviation from random movement could result in the formation of hubs, which are neighborhoods in the mobility network that receive a disproportionate share of a city's daily trips. In 
the context of a city, these places tend to be downtown areas or central business districts. The second dimension of inequality in mobility, and perhaps the more important one for understanding the inequality arising as a result of how people move, is the ways in which disadvantaged places are disproportionately connected to one another. This captures the degree to which neighborhood mobility networks are isolated from one another, or how clustered the mobility network is. Recent research on mobility inequality has found that such clustering is common in U.S. cities but that significant heterogeneity exists across contexts (Phillips et al. 2019; Wang et al. 2018).

Inequality in mobility patterns implies differential exposure to a range of physical and social neighborhood contexts, which are consequential. As neighborhood contexts matter for a vast array of social and health outcomes (Chetty, Hendren, and Katz 2016; Sharkey and Faber 2014) and neighborhoods have stronger mobility connections to neighborhoods similar to themselves (Krivo et al. 2013; Wang et al. 2018), researchers have hypothesized that mobility inequality works to further spatially concentrate disadvantage (Levy et al. 2020). Research on health exposures, crime, and even occupational opportunities supports this hypothesis. For example, daily locations outside the home have been connected to various health outcomes (Cagney et al. 2020; Chaix 2009; Sharp, Denney, and Kimbro 2015). Epidemiologists using GPS data found that patterns of movement in cities expose individuals differentially to environmental contaminants like air pollution that are important factors in many chronic diseases (Kim and Kwan 2021; Ma et al. 2020; Perchoux et al. 2013). Where people travel during the day also has the potential to affect their economic trajectory. Sugie and Lens (2017) found evidence of a spatial mismatch between where a person lives and travels daily and their occupational opportunities. Studying newly released prisoners looking for work, they documented that those who spent time during the day in places with more economic opportunities tended to have better success securing employment. Recent work on crime has similarly found connections between where people spend time and their exposure to crime, as well as the spread of violent crime across neighborhoods (Levy et al. 2020; Papachristos et al. 2011; Browning et al. 2021). For example, Levy and colleagues documented that network disadvantage, measured as inequalities in both the places visited and visitors' origin, interacted with neighborhood disadvantage and predicted the spread of violent crime across a city. Last but not least, mobility networks are important for the transmission of disease (Balcan et al. 2009), including COVID-19 (Badr et al. 2020; Chang et al. 2021; Glaeser et al. 2020; Nouvellet et al. 2021). However, mobility inequality further suggests that disease progression will at least initially concentrate in some areas over others because contact networks formed from travel throughout a city will be socially stratified. In a simulation of COVID-19 spread in 10 U.S. cities using observed mobility networks, Chang and colleagues (2021) found that the predicted outcomes reflected observed inequalities in case rates between racial and ethnic groups.

Despite the growing literature establishing the importance of mobility inequality in social stratification, we still know relatively little about how it might change over time and how the disruptions of the COVID-19 pandemic in 2020 may have affected it. The pandemic is a ripe time to explore this topic because many of the policies 
aimed at slowing the spread of COVID-19 in urban spaces were tremendously disruptive to mobility. Stay-at-home orders, business closures, limitations on public transportation, shifts to remote work and education, and finally, social distancing guidelines that shrank the size of social networks (Feehan and Mahmud 2021; Weeden and Cornwell 2020) all have the potential to change mobility between neighborhoods and therefore the levels of mobility inequality. Evidence is yet to emerge on whether these changes are short-lived or long-lasting. We use the COVID-19 pandemic as an opportunity to better understand the dynamics of mobility inequality in cities in the face of crisis and disruption.

We make three contributions to the urban sociological literature on mobility network inequality and neighborhood isolation, along with research on the societal impacts of COVID-19. First, we depart from previous studies that focused on mobility inequality at a single point in time. We extend this work by calculating weekly values of network inequality in 2019 and 2020. Describing network inequality over time also allows us to illustrate how mobility networks responded to the COVID-19 pandemic in 2020. Finally, we contribute to the growing literature connecting urban mobility to the COVID-19 pandemic by estimating a linear regression model with week and city fixed effects to assess the relationship between change in mobility networks and the new COVID-19 cases the week before.

\section{Data and Methods}

We use three main sources of data: mobility data, COVID-19 case counts, and demographic composition and other metrics of places. We discuss these sources and the measure we create from each in turn.

\section{Mobility Data}

Mobility data come from the SafeGraph Social Distancing Metrics data set, which includes daily counts of trips between pairs of U.S. block groups in 2019 and 2020 (SafeGraph 2021a). SafeGraph data track the mobility of a large sample of U.S.-based smartphones. User information is anonymized and aggregated, and additional noise is introduced on aggregate values to protect users' identities in sparsely populated areas. SafeGraph determines the home block group by identifying a device's most common nighttime location over six weeks (SafeGraph 2021b). In 2020, SafeGraph made the Social Distancing Metrics data publicly available to researchers to promote work on the effects of COVID-19 pandemic, and it has been used extensively since (Chang et al. 2021; Gao et al. 2020; Glaeser et al. 2020; Gupta et al. 2020). Other large sources of mobility data include proprietary cell phone-based data sets (Prestby et al. 2020), geo-coded Twitter data (Levy et al. 2020; Phillips et al. 2019; Wang et al. 2018), and mobility pattern data sets from Apple and Google (Nouvellet et al. 2021). However, the combination of the ease of access of the SafeGraph data set, its geographic resolution, and its daily measurement makes these data best suited for our questions about weekly changes in neighborhood level mobility networks. 
In the mobility networks we study, nodes are census tracts, and we base the measures we compute on this network. We recognize that neighborhoods and tracts are not analogous geographic units, yet we use this terminology for ease of presentation. To construct a network of census tracts that are connected by people moving between them, we begin with block group level data. For each block group, SafeGraph reports a count of observed trips to all other census block groups. We then aggregate trips to represent travel between tracts. Finally, for each tract, we convert the count of trips in its ego-network to represent the proportion of the total trips from a tract to every other tract. Therefore, the weighted edges of the mobility network are the proportion of the total weekly travel from a tract allocated to each destination tract. We eliminate trips to tracts outside of the city boundaries (specifically, the counties within which cities lie) and trips within the home census tract, so our results are reporting within-place inequality in travel outside of the home neighborhood.

As with any cell phone-based data set, there are questions of demographic representativeness that extend to the SafeGraph data. Surveys of smartphone ownership indicate potential under-representation of low-income individuals and individuals aged more than 65 and over-representation of higher-income groups (Pew Research Center 2021). At the population level and in large spatial units, however, analysis of the SafeGraph data indicates relatively low levels of demographic bias (Squire 2019). In contrast, at smaller units such as individual points of interest, researchers have found evidence of age, race, and ethnicity biases in the mobility data (Coston et al. 2021).

The key concern for our research on mobility inequality is that if under- or over-represented groups differ significantly in their daily movement patterns, then biases in our measures could emerge. For example, suppose the sample misses large numbers of individuals who travel daily to the downtown area of a city for work. In this case, we would underestimate the importance of hubs in the mobility network. Similarly, if an over-represented group travels to more neighborhoods similar to their own than most, then we could underestimate neighborhood isolation. We do not have a strong sense of the direction of the possible biases. However, by aggregating our data to tracts and computing city level index measures based on the distribution of travel rather than its volume, any biases created by demographic characteristics at smaller spatial units are likely to be significantly mitigated.

Nevertheless, we do not overemphasize individual city estimates of our index measures or slight differences in cities' relative positions. Instead, our research questions leverage an important strength of the SafeGraph data: their temporal resolution. SafeGraph provides daily estimates of mobility, which we aggregate to weekly averages. This allows us to compare across cities and to evaluate broad patterns of change in the observed mobility patterns. Furthermore, our regression analysis includes city effects, which help control for differences in possible sample bias across cities. 


\section{COVID-19 Case Data}

We extracted COVID-19 case data from the New York Times database reporting daily case counts in U.S. counties (New York Times 2021). The data begin with the first known U.S. cases in January 2020 and continue through the year. These data are scraped from publicly reported figures by county health departments and published daily online. The key measure we consider from these data is the number of new cases in a county detected the previous week, which we assume might influence how much people move and where they go (Shamshiripour et al. 2020).

\section{Demographic Composition and Other Place-Based Data}

In addition to case and mobility data, we include a number of county level characteristics from the U.S. Census Bureau's American Community Survey, 2014 to 2018 estimates. The included variables follow the models used by Phillips and colleagues in their original study of cross-city variation in equitable mobility index (EMI) scores (2019). First, to account for variation in the size of a place, which may make integration of places within a mobility network more difficult, we include the population and area in square meters. Next, we include the percentage of the population that commutes to work via public transportation. Although in their original study, Phillips and colleagues found that public transportation was not statistically significantly related to variation in EMI between cities, public transportation is potentially important for understanding changes during the pandemic. Public transportation closures, restrictions, and rider hesitancy have meant that ridership declined during the pandemic (Liu, Miller, and Scheff 2020) and therefore has the potential to alter inequality in mobility networks.

Next, Phillips and colleagues hypothesized that more diverse and highly educated places would have less mobility inequality because residents are less likely to avoid neighborhoods with compositions more dissimilar to their own. This idea is captured using the percentage of the population with a bachelor's degree or higher and Blau's diversity index. The diversity index is based on the proportions of seven racial and ethnic groups: white, black, Hispanic, Asian, Native Hawaiian and other Pacific Islander, American Indian and Alaska Native, and other. Finally, residential segregation at the neighborhood level is a well-known facet of American cities that Phillips and colleagues found was related to mobility inequality. Places with higher levels of residential segregation on average had more neighborhood isolation. Inspired by these findings, we include measures of racial and income segregation to see if they are associated with changes in mobility inequality. We calculate Theil's information index ( $\mathrm{H}$ index) to measure both income and racial segregation using the segregation package in $\mathrm{R}$ (Elbers 2021). The $\mathrm{H}$ index of income is based on the 16 categories of income provided by the census. The $\mathrm{H}$ index of race is based on the same seven categories used for the calculation of Blau's diversity index. Table 1 presents summary statistics for the variables used in our regression analysis. 
Table 1: Summary statistics.

\begin{tabular}{lrrrr}
\hline & Mean & SD & Min & Max \\
\hline Relative EMI & -0.02 & 0.01 & -0.08 & 0.01 \\
New cases (thousands) & 3.03 & 5.48 & 0.00 & 77.49 \\
Population (100 thousand) & 25.07 & 24.27 & 6.84 & 101.98 \\
Area (square kilometers) & 310.49 & $5,188.27$ & 127.26 & $23,889.46$ \\
Public transportation (\%) & 5.44 & 7.29 & 0.23 & 26.33 \\
Bachelor's degree (\%) & 15.69 & 4.20 & 9.31 & 27.19 \\
Blau heterogeneity & 0.63 & 0.08 & 0.31 & 0.74 \\
H index for race & 0.25 & 0.08 & 0.14 & 0.49 \\
H index for income & 0.08 & 0.01 & 0.06 & 0.09 \\
\hline
\end{tabular}

Note: Data from the New York Times COVID-19 database and the American Community Survey, 2014 to 2018. SD, standard deviation.

\section{Measuring Mobility Inequality}

We closely follow the approach of Phillips and colleagues, who developed two indices to characterize mobility networks' evenness and concentration (2019). These indices measure two different dimensions of mobility networks. The first, the concentrated mobility index (CMI), measures the importance of hubs in the overall network. The CMI is calculated as one minus the Gini coefficient of the indegree distribution, which itself is calculated by summing the weighted edges of the network. Therefore, CMI ranges from 0 to 1 , with lower values representing more even degree distributions within the network and higher values representing network unevenness or the presence of hubs. Intuitively, a mobility hub in a city is a downtown area or a central business district. As CMI approaches 1, larger proportions of travel is directed through fewer locations. The second index developed by Phillips and colleagues is the EMI. The EMI measures how isolated neighborhood mobility networks are from one another. The EMI is calculated by comparing the observed mobility network with a network where people from all neighborhoods visit one another at the same rate. The difference between the two is quantified using Hamming distance (HD) and scaled to fit between 0 and 1 by dividing by the theoretical maximum Hamming distance in the network. See Equations (2) and (3) in the original work of Phillip and colleagues for full explanation of calculating the Hamming distance and its maximum value, specifically,

$$
\mathrm{EMI}=1-\frac{\mathrm{HD}_{\text {Observed }}}{\mathrm{HD}_{\text {Maximum }}}
$$

In contrast to the CMI, lower values of the EMI represent more neighborhood isolation, that is, mobility within clusters of a network rather that between clusters. As EMI approaches zero, there is less overlap in neighborhood mobility networks, and thus places are more isolated from one another. Phillips and colleagues found that EMI scores tended to decrease as the size and population of a city increase. Additionally, as measures of racial and economic segregation increase, neighborhood isolation measured by EMI also increase (Phillips et al. 2019). 
We calculate weekly EMI and CMI values in 2019 and 2020 for counties containing the 25 largest cities in the United States. Because our interest is in changes to these scores, we also calculate the relative EMI and CMI by subtracting 2020 values from each city's 2019 average. Note that this difference is zero if the averages do not differ; negative values express a less hub-like mobility network structure in the CMI and more modular structure in the EMI. We use counties rather than the municipality boundaries of cities as this way our place unit matches the COVID-19 data reported by county health departments. The New York City and Oklahoma City municipal boundaries cross county lines, and in these cases, we combine counties into a single place. Our focus on counties rather than city boundaries has the benefit of matching the available COVID-19 data, but it does introduce some uncertainty in the comparability between places. For example, whereas the New York City boundary coincides with the counties it comprises, many counties have suburban or even rural areas beyond the dominant city's boundary. Although this issue represents a shortcoming of our approach, our focus on changes within rather than between places means this issue is not as critical as it would be for other research designs focusing on between-city comparisons.

\section{Results}

Figure 1 presents the 2019 and 2020 weekly CMI and EMI scores for all 25 cities in our sample. Whereas Phillips and colleagues have considered these indices as a single measure for cities, we show how they evolve over time. We find that in 2019 both CMI and EMI are stable across all cities. Specifically, CMI values fluctuated by only 2.7 to 6.4 percentage points, whereas EMI values changed by only 0.5 to 2.9 percentage points. Thus, even small deviations in 2020 could represent a meaningful change in the structure of mobility networks. With this in mind, it is immediately apparent how disruptive the pandemic in 2020 was for many cities' mobility networks. Even at this scale, almost every city saw some noticeable change during 2020. In Figure 1 of the the online supplement, we also include an illustration of the mobility network in four cities at four different time points to visually substantiate this point.

To get a better sense of the patterns of change across all the cities, we turn to Figure 2, which presents weekly values of CMI relative to a city's 2019 average. Each line in Figure 2 represents a single city, and the red line is the weekly average CMI across all cities. Beginning in the first weeks of March and continuing into April, there is a sharp decline in CMI across all cities. For example, in the week of April 8, the largest decrease in CMI was -0.17 in San Francisco, and the smallest occurred in Houston with a value of -0.004 . Smaller values of CMI indicate a decrease in how important hubs have been in a mobility network. These results are further supported by looking at the inverse cumulative distribution function of indegree over time (see Figure 2 of the online supplement). Thus, the observed decline in CMI suggests that downtown or other mobility hubs in cities diminished in their importance. The decline in centrality of business districts makes intuitive sense given that many cities opted to close nonessential businesses and many occupations saw a potentially permanent move to remote work (Brynjolfsson et al. 

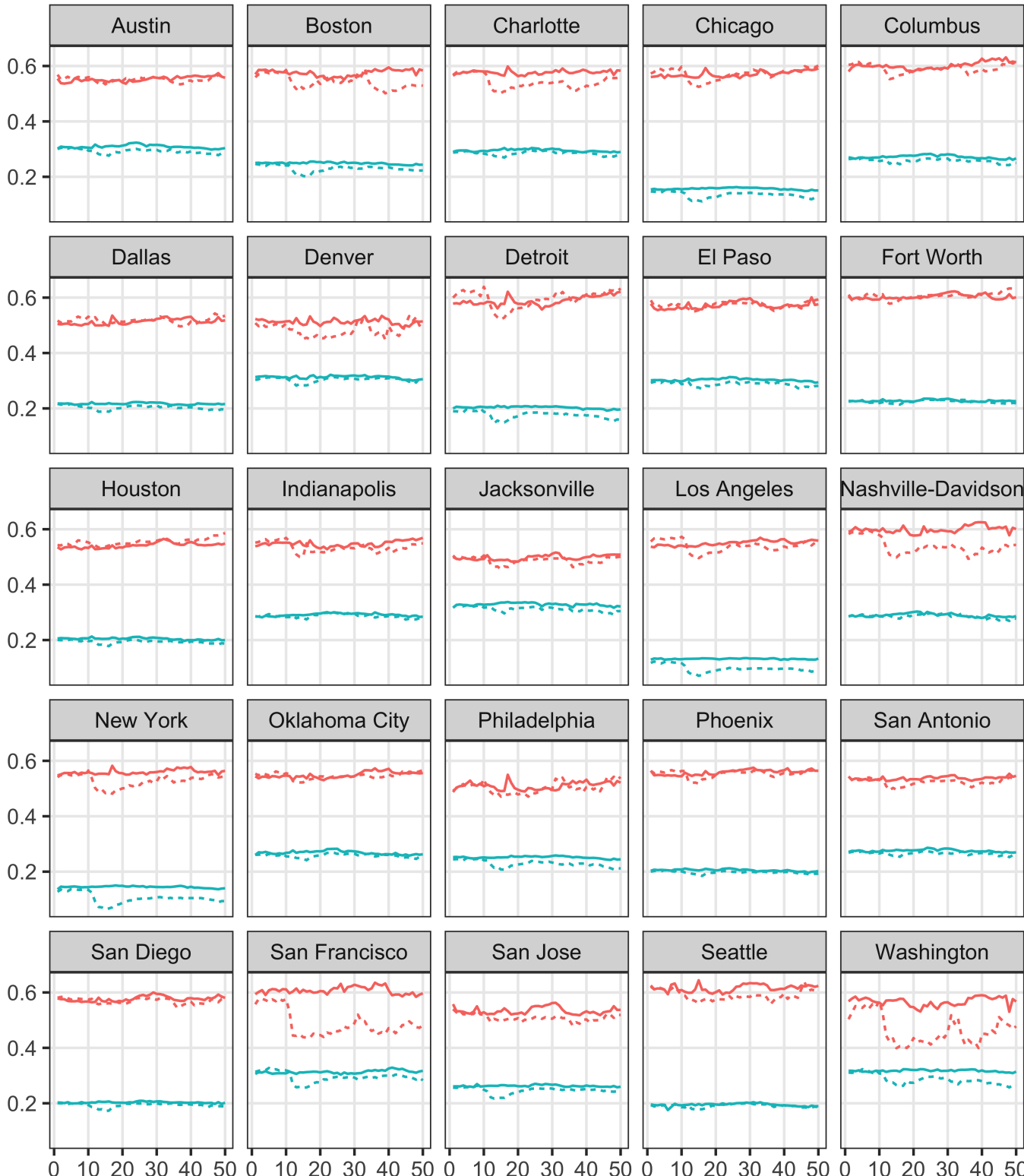

week
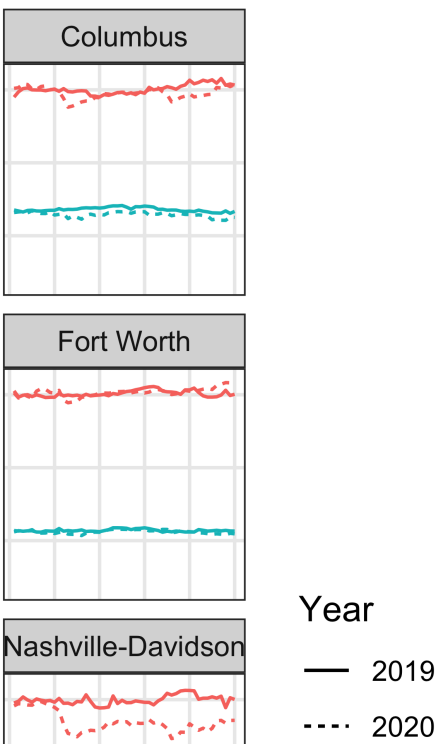

Measure

- $\mathrm{CMI}$

- EMI

Figure 1: Weekly CMI and EMI values in the 25 largest U.S. cities.

2020). However, what is surprising is the uniformity in response across cities despite the varying states of the COVID-19 pandemic and mandatory restrictions to mobility in each during the months of March and April. Not every city closed businesses in mid-March, but almost every city in our sample saw a downward shift in its CMI during this time. By the end of April, CMI values began to return to their 2019 averages in most places, and differences were generally within the ranges of 2019 values. There are four exceptions to this observation: Boston, Nashville, 


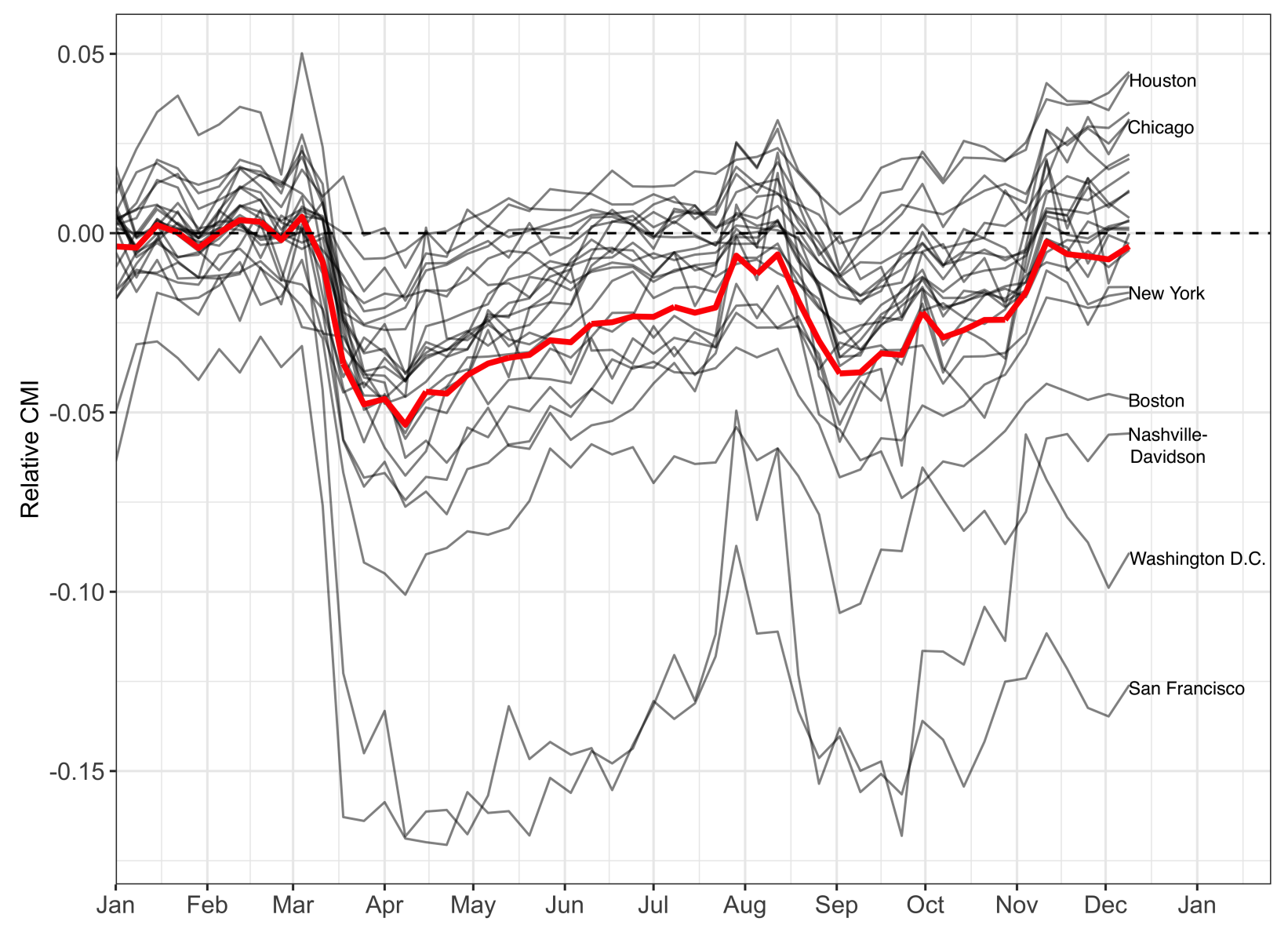

Figure 2: Weekly CMI relative to 2019 average. Each line represents changes in a single city, and the red line is the weekly average.

San Francisco, and Washington, DC. In these four cities, mobility hubs declined in importance, and CMIs remained well below their 2019 averages for the remainder of 2020.

Moving on to EMI, Figure 3 presents the weekly EMI scores relative to a city's 2019 average. Overall, there is less variation in EMI than CMI, but the pattern of change is more consistent across cities in EMI. Like CMI, EMI exhibits a sharp decline starting in mid-March and continuing into April. Unlike CMI, lower scores of EMI indicate an increase in neighborhood isolation and therefore mobility inequality. Therefore, Figure 3 detects significant increases in neighborhood isolation. During May and the subsequent summer months, EMI values trended upward toward 2019 averages. However, many cities never fully returned to their prepandemic values, and in the fall months, EMI values uniformly trended downward again. We conclude from Figure 3 that the 2020 pandemic significantly affected neighborhood isolation measured by EMI for the cities in our sample. Furthermore, few cities returned to their baseline mobility patterns by the end of 2020 . 


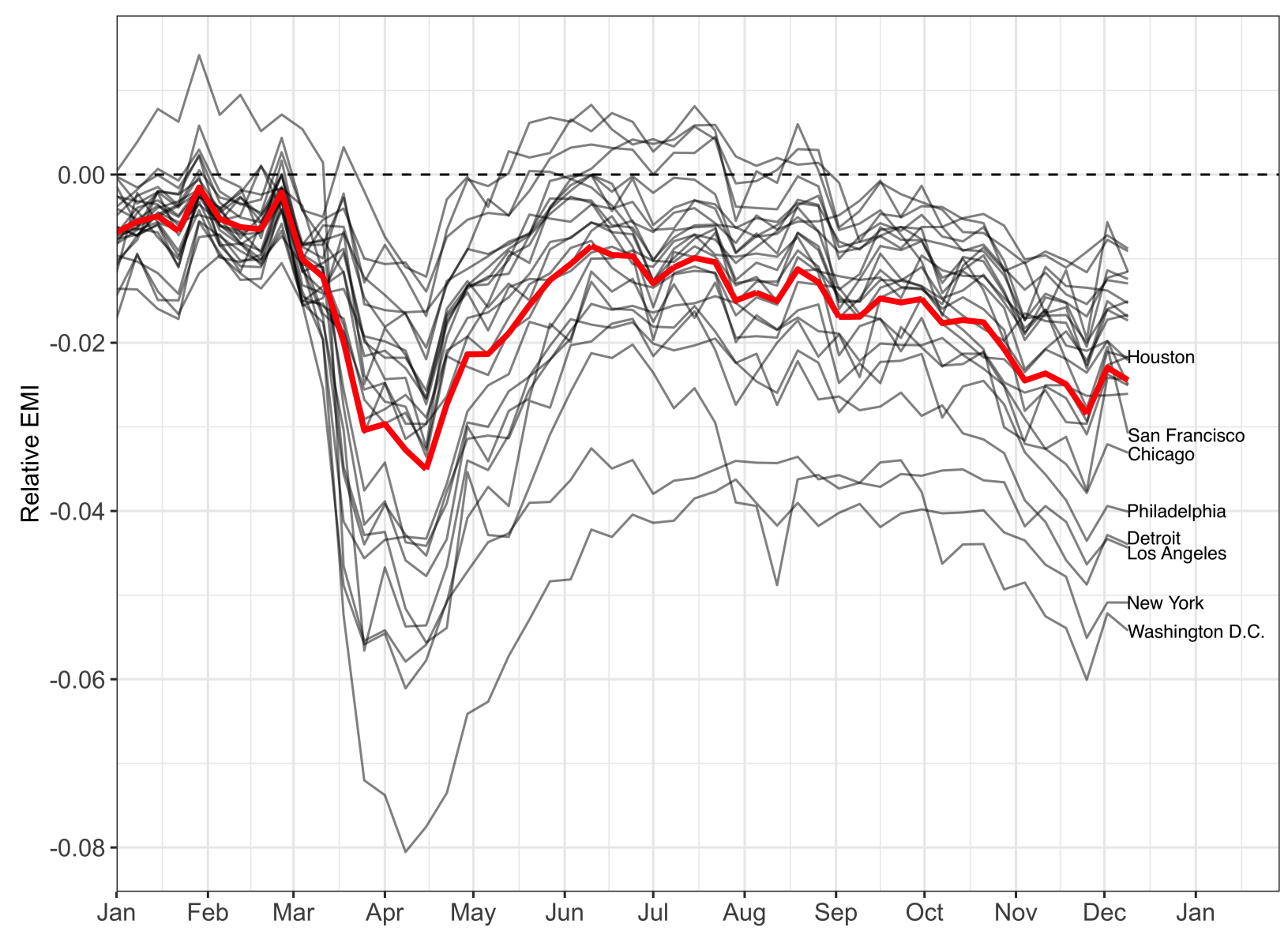

Figure 3: Weekly EMI relative to 2019 average. Each line represents changes in a single city, and the red line is the weekly average.

Both trends in CMI and EMI are related to the overall decline in mobility in cities during the pandemic. However, mobility decline alone generally cannot explain our observations. For example, if mobility decline were uniform for all neighborhoods, then the relative importance of hubs, which influences CMI, and the proportion of travel to other neighborhoods, which influences EMI, would remain the same. Thus, overall declines in mobility are important, but it is the disparate patterns of decline along the edges of the mobility network that produces changes in CMI and EMI.

In general, EMI and CMI exhibit similar patterns of change in 2020. First, there was a noticeable decline in values starting in early March and continuing into April, followed by regression to 2019 values, with some cities never reaching them. Additionally, EMI appears to decline from September onward. This second decline in EMI coincides with the timing of the second wave of cases in the United States. This suggests that EMI, unlike CMI, might be continuously responsive to the pandemic conditions within a city. 
To better understand the relationship between EMI and COVID-19 cases, we estimate a mixed effects regression model with city and week effects (see Table 2). Our response variable is the change in a city's EMI relative to its 2019 average. We treat cities as unconnected to one another so that our observations are the city-weeks in which we have case data. For each city ( 25 cities) we have a weekly ( 40 weeks) repeated measure of relative EMI. We limit our data to begin March 11, the week when new case data are available for all weeks for a total of 1,000 observations. Week effects are also important to our model because they help control for seasonality and weekly changes in the attitudes toward the pandemic nationwide. To assess the correlation between new cases and mobility, we lag new cases by a week. Therefore, in our regression models, changes in the dependent variable are predicted by the number of new cases in the prior week. All variables have been standardized so that the units of all coefficients are standard deviations.

We find a small but significant relationship between new cases and changes in EMI a week later. On average, after controlling for city and week effects, every standard deviation $(5,480$ cases) increase in new cases within a city produces a 0.1 percent (standard error [se] $=0.02, p<0.01$ ) decline in EMI. For perspective, Los Angeles had the largest weekly total of new cases at 77,490 cases during the week beginning with December 2. Our model, therefore, predicts a 1.4 percent decline in EMI as a result of new cases alone. For the same week in Los Angeles, our full model predicts a 5.5 percent decline in EMI, which means that new cases account for 25 percent of the predicted decline. Other cities had, on average, just more than 13,400 cases at their peak case count. In the average pandemic week, our model predicts a 0.2 percent decline in EMI as a result of new cases. In sum, our findings suggest that mobility inequality is related positively to new cases in a city.

In our second model, we include additional place characteristics calculated from the U.S. Census Bureau data. The area of a place in square meters had no statistically significant relationship to the relative EMI. However, a standard deviation increase in population produced, on average, a 0.4 percent (se $=0.20, p<0.10$ ) decline in EMI relative to 2019. Thus, places with larger populations, regardless of the area of the place, saw declines in EMI during 2020. Neither the percentage of the population with a bachelor's degree nor Blau's heterogeneity index had a meaningful relationship to the change in EMI relative to 2019. Our model predicts a very small and insignificant relationship between income segregation and relative EMI; however, segregation by race and ethnicity appear important. For every standard deviation change in $\mathrm{H}$ index for race, EMI declined by 0.4 percent (se $=0.2, p<0.05$ ).

Finally, among our predictors, the percentage of the population using public transportation to commute to work had the strongest relationship with changes in EMI in 2020. For every standard deviation change in the percentage of the population using public transportation, EMI declined on average by 0.6 percent (se $=0.2$, $p<0.001$ ). This association stands out because in their original study, Phillips and colleagues found no statistically significant relationship between public transportation use and absolute EMI between cities. However, our finding suggests that within a city, public transportation is important for decreasing mobility inequality and that when its use declines, neighborhood isolation tends to increase. 
Table 2: Mixed effects models of relative equitable mobility index.

\begin{tabular}{lrr}
\hline & $(1)$ & $(2)$ \\
\hline New cases (1 week lag) & $-0.001^{\dagger}$ & $-0.001^{\dagger}$ \\
Population & $(0.000)$ & $(0.000)$ \\
& & $-0.004^{*}$ \\
Area (square meters) & $(0.002)$ \\
Public transportation (\%) & 0.001 \\
Bachelor's degree (\%) & $(0.002)$ \\
H index for income & $-0.006^{\dagger}$ \\
H index for race & $(0.002)$ \\
Blau heterogeneity & & 0.0001 \\
& & $(0.002)$ \\
Constant & & 0.0001 \\
& & $(0.002)$ \\
\hline Log likelihood & & $-0.004^{*}$ \\
Akaike information criterion & & $0.002)$ \\
Bayesian information criterion & $-0.018^{\dagger}$ & $(0.002)$ \\
\hline
\end{tabular}

Note: All coefficients are standardized. 1,000 observations. $+p<0.01 ; * p<0.05$.

Our results looking at changes in EMI, along with the regression models finding a relationship between those changes and racial segregation, prompt one final analysis to see if we can better understand how changes in mobility patterns between different types of places occurred during the pandemic. For this, we categorized each census tract in our city mobility networks according to their racial, ethnic, and class composition. We classify tracts as poor if more than 30 percent of the population is below the federal poverty line. Second, we labeled tracts by their majority racial or ethnic group based on a 50 percent threshold. Tracts with no majority racial or ethnic group are labeled "mixed." We combine these two classifications to create 10 groups. Finally, we aggregate mobility data based on the categorization of both the sending and receiving census tracts and compare the changes between 2019 and 2020 mobility as a change in the percentage of 2019 trips. Our goal is to detect differences in mobility between census tracts that belong to these 10 categories during the pandemic and, in particular, to characterize any change in neighborhood mobility networks.

Figure 4 presents our results of changes between categories for the week of April 7 in a sample of four cities. Each cell represents the percent change in trips between 2019 in 2020. Missing cells indicate that either the tract category is not present in a city or there is no connection between those tract types. Furthermore, the columns of Figure 4 vary based on three selection criteria that limit trips according to their spatial proximity. The first column includes all trips between places. Notably, this 


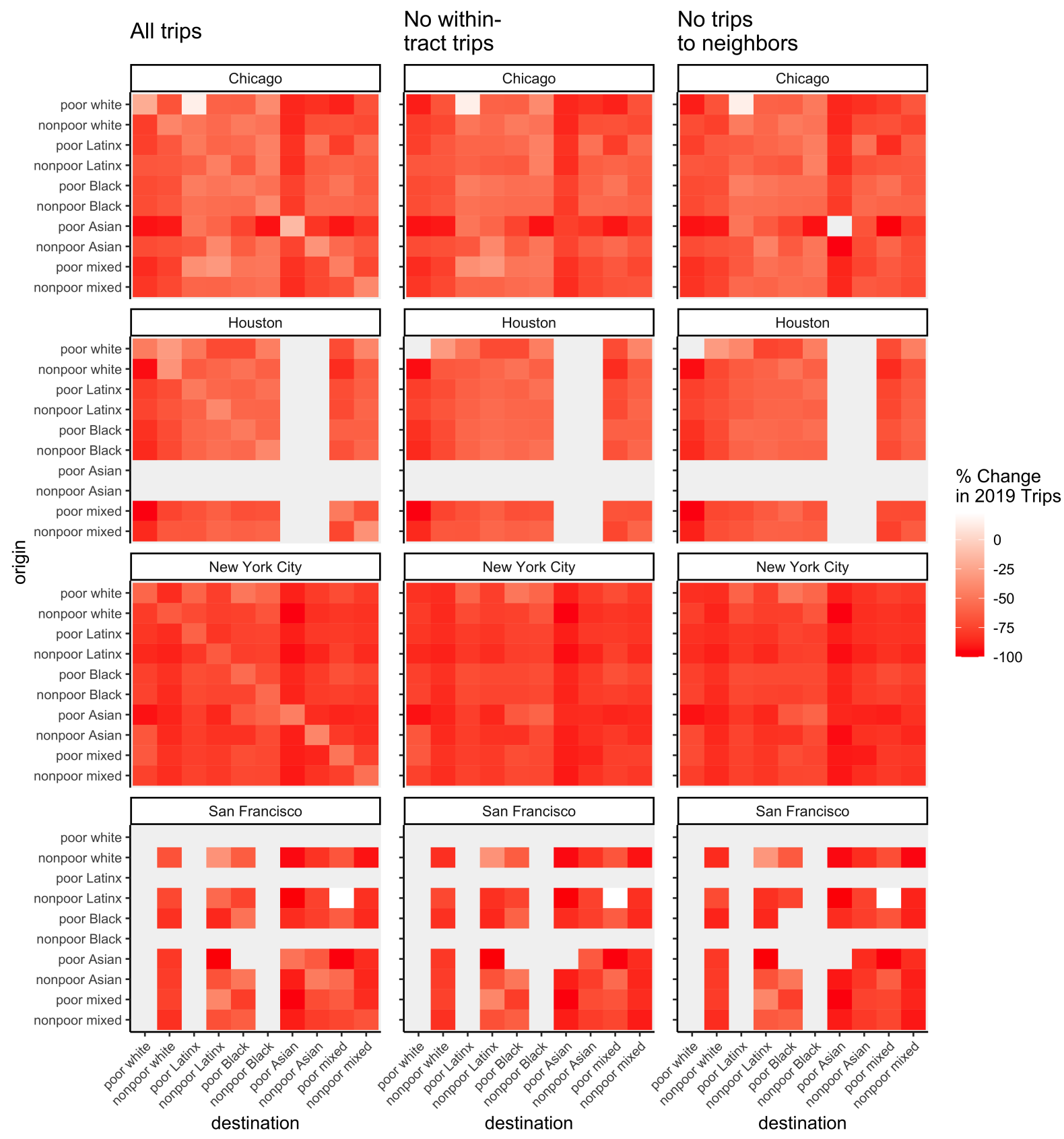

Figure 4: Change in trips between 2019 and 2020 as a percentage of 2019 trips. Each row is a different origin category, and each column is a destination category. Cells are shaded according to their percent change in mobility relative to in 2019 values. The left column includes all trips in our data. The middle column removes trips within the home census tract, and the rightmost column removes trips both from within the home census tract and to immediately adjacent tracts.

includes trips out of the home but within the home census tract. This selection differs from our calculation of EMI, which excluded within-tract trips consistent 
with the approach of Phillips and colleagues (2019). The second column removes within-tract trips, and the third column removes trips to neighboring census tracts.

The first observation we highlight from Figure 4 is that in almost all cases, mobility declined significantly between places. In some cases, that decline was nearing 100 percent, meaning there were no recorded trips in 2020. Importantly, however, the decline was not uniform across all places. Of particular interest in Figure 4 are the trips between places of the same type, which are on the matrix's diagonal. In the leftmost column, the diagonals of all four cities are noticeably lighter in color. This pattern indicates that trips between places of the same category declined less than trips to other categories of places. This finding is evidence that during the pandemic, not only did neighborhood mobility become more modular, as changes in the EMI have revealed, but also isolation was stratified along race and class lines. This finding holds across all groups in our data. However, one other change stands out as well. In April of 2020, trips to and from poor majority Asian tracts decline more relative to other tract categories. This significant decline is evidence that people, regardless of their origin census tract category, avoided traveling to majority Asian neighborhoods. In the weeks before the data shown here, President Trump and other prominent political leaders in the United States began referring to COVID-19 as the "Chinese flu" and blaming China for spreading the virus globally (He et al. 2020; Reny and Barreto 2020; Rizzuto 2020; Tavernise and Oppel, Jr. 2020). The consequences of this fearmongering may be seen in our data, which suggest that people in many cities avoided predominately Asian neighborhoods by April.

In columns two and three, we remove within-tract trips and trips to neighboring census tracts to better understand the spatial stratification of change in mobility we observe. In particular, we were interested in whether it could be occurring because people were geographically limiting their travel to more proximate neighborhoods. After we remove within-tract trips (i.e., the shortest trips), the diagonal disappears, suggesting no strong differences between similar and different places in mobility decline. This pattern remains true when the shortest sets of trips and trips to immediate neighbors are also removed in column three. The two columns are visually difficult to tell apart, showing us that most of the decline between places occurs at distances beyond the home neighborhood's immediate vicinity. Figure 5 and Table 1 in the online supplement confirm this observation, illustrating that the distribution of travel distances changed markedly between 2019 and 2020. In fact, in 2019, trips within the home census tract represented 78 percent of all trips in our data. By April 2020, trips outside of the home census tract declined so that 90 percent of all travel was within the home census tract. Even when people ventured beyond their home census tract, trips tended to be slightly more concentrated at shorter distances. In sum, in 2020, travel declined from places outside the vicinity of one's immediate neighborhood and toward trips within the home census tract. Overall, these figures suggests that people's everyday mobility was more geographically constrained and more isolated from places dissimilar to their home census tract. 


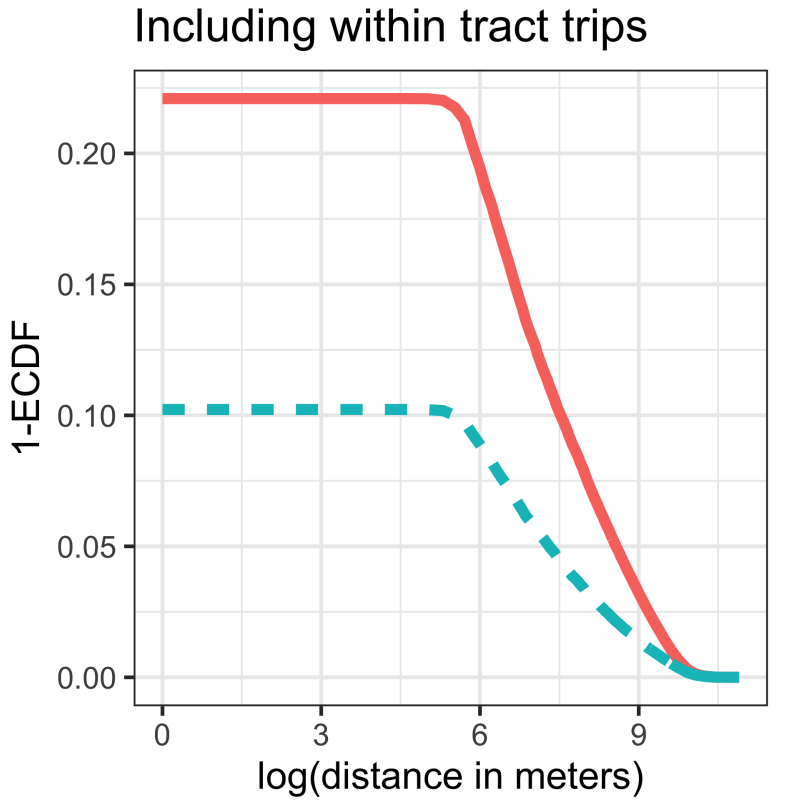

Year $2019=$ | 2020

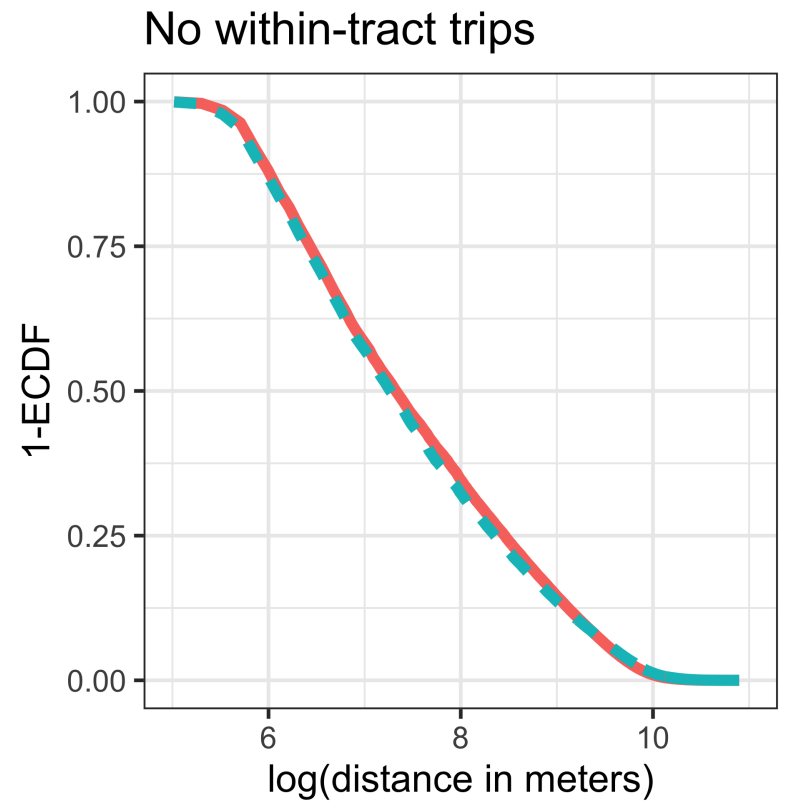

Year $2019=\mid 2020$

Figure 5: Complementary empirical cumulative distribution function (1-ECDF) of trip distances of tracts during the week of April 7 in New York City. Trips within the home census tract are given a distance of zero.

\section{Robustness Analyses}

The choice of spatial unit was our primary source of concern in terms of the robustness of our results. Accordingly, we explored two alternative specifications to assess the sensitivity of our analysis to these choices. First, we repeated our descriptive analyses using ZIP codes as nodes as opposed the smaller census tracts. Our results (see Figure 3 of the online supplement) were consistent for analysis of EMI. However, CMI no longer exhibits a clear pattern across all cities. Although a handful of cities do display the same decline in CMI during the end of April, the pattern is no longer obvious across all of the cities.

Second, we chose counties as the boundaries of our places rather than municipal boundaries. This decision allows us to model the relationship between COVID-19 cases and relative EMI, but it introduces some uncertainty because some county boundaries include additional suburban or rural areas. The additional areas around a county could bias our results toward being more unequal by adding neighborhoods not integrated or meaningfully a part of a city's mobility network. To assess the sensitivity of our results to this decision, we repeated the descriptive analyses, this time with a selection of the top 10 largest cities and using their municipal boundaries instead of the county boundaries. The results were again consistent with our headline findings regarding trends in CMI and EMI during 2020 (see Figure 4 of the online supplement). 


\section{Conclusion}

Using two mobility inequality measures we find evidence that the COVID-19 pandemic induced changes in mobility inequality in 2020 in the 25 largest cities in the United States. In one way, inequality declined during the pandemic, as the importance of downtown hubs decreased in the earliest phases of the pandemic. This suggests that mobility networks have generally become more even between neighborhoods. However, after the initial shock, no consistent pattern remained, as many cities returned to their 2019 baselines and others even increased inequality in terms of the importance of hubs.

On the other hand, a measures of neighborhood isolation (EMI) showed a clear and consistent pattern of elevated neighborhood isolation across the 25 largest cities. All cities in our sample saw increases in neighborhood isolation starting in mid-March. This increase in isolation continued until the first weeks of April, when cities had an average decline in EMI of 3.2 percent and a maximum decline of more than 8 percent. The timing of the initial sharp increase in neighborhood isolation suggests that some combination of national attention to the spread of COVID-19 stoked by the declaration of a national emergency in mid-March, along with strict mobility restrictions in important municipalities like New York City and Chicago, caused a nationwide response, even in places where no municipal restrictions to mobility had been introduced. After April, inequality began to return to its 2019 levels, but only two cities returned completely. In fact, in the last months of 2020, as a nationwide surge in cases developed, neighborhood isolation trended upward yet again. Furthermore, we find that regardless of the weekly conditions in a city, an increase of new cases in the previous week correlates with a decline in EMI, that is, an increase in neighborhood isolation. Place characteristics also contributed to decline in EMI during the same period. In our models, the population's size, population proportion using public transportation, and the $\mathrm{H}$ index for racial segregation had measurably significant effects on EMI changes. Other place characteristics such as the area, percentage of the population with a bachelor's degree or higher, $\mathrm{H}$ index for income segregation, and Blau's heterogeneity index had comparatively small and insignificant effects.

Future research should continue to explore how sociospatial stratification is related to our findings of increased neighborhood isolation. Our results indicate that neighborhoods became more isolated from one another and potentially have reached a new steady state. Our results suggest that mobility isolation also means a decline in contact between socially dissimilar neighborhoods. This finding, especially our finding that poor majority Asian tracts became very isolated, could have significant ramifications for intergroup relationships in cities (Pettigrew 1998). For example, contact and group threat theory posit that regular interactions between groups are essential for minimizing prejudice. However, if daily mobility changes also mean less contact between groups, these positive outcomes in cities may be at risk. Intergroup relations in the United States are already a growing area of concern for many Americans (Newport 2020); therefore, we should be particularly attuned to any changes in the amount and type of contact between people of different ethnic and racial backgrounds during their routine daily activities. Additionally, mobility 
isolation could intensify neighborhood disadvantage by limiting access to resources most needed in resource-poor neighborhoods. For example, social networks across places are essential to understand where people search for housing and jobs. These patterns, in turn, contribute to the creation and maintenance of residential segregation (Krysan and Crowder 2017) and feed into other forms of inequality, such as by income. Thus, more isolation worryingly suggests less intergroup contact and even further disadvantage in terms of access to resources for some communities.

For now, our results indicate that the COVID-19 pandemic directly affected patterns of mobility in cities. These impacts include a decline in the importance of downtown areas and increased neighborhood isolation. Even more worrisome is that few cities returned to pre-pandemic mobility patterns even after mobility restrictions ended, and with new daily routines solidifying, these could be signs for a new equilibrium.

\section{References}

Badr, Hamada S., Hongru Du, Maximilian Marshall, Ensheng Dong, Marietta M. Squire, and Lauren M. Gardner. 2020. “Association between Mobility Patterns and COVID-19 Transmission in the USA: A Mathematical Modelling Study." Lancet Infectious Diseases 20(11):1247-54. https : //doi .org/10 .1016/S1473-3099(20)30553-3.

Balcan, Duygu, Vittoria Colizza, Bruno Gonçalves, Hao Hu, José J. Ramasco, and Alessandro Vespignani. 2009. "Multiscale Mobility Networks and the Spatial Spreading of Infectious Diseases." Proceedings of the National Academy of Sciences 106(51):21484-89. https://doi. org/10.1073/pnas.0906910106.

Browning, Christopher R., Nicolo P. Pinchak, and Catherine A. Calder. 2021. "Human Mobility and Crime: Theoretical Approaches and Novel Data Collection Strategies." Annual Review of Criminology 4:99-123. https : //doi .org/10 .1146/annurev-criminol-061020021551.

Brynjolfsson, Erik, John J. Horton, Adam Ozimek, Daniel Rock, Garima Sharma, and Hong-Yi TuYe. 2020. "COVID-19 and Remote Work: An Early Look at US Data." Technical Report 27344, National Bureau of Economic Research.

Cagney, Kathleen A., Erin York Cornwell, Alyssa W. Goldman, and Liang Cai. 2020. “Urban Mobility and Activity Space." Annual Review of Sociology 46:623-48. https ://doi .org/10. 1146/annurev-soc-121919-054848.

Chaix, Basile. 2009. “Geographic Life Environments and Coronary Heart Disease: A Literature Review, Theoretical Contributions, Methodological Updates, and a Research Agenda." Annual Review of Public Health 30:81-105. https : //doi . org/10.1146/annurev . publhealth.031308.100158.

Chang, Serina, Emma Pierson, Pang Wei Koh, Jaline Gerardin, Beth Redbird, David Grusky, and Jure Leskovec. 2021. "Mobility Network Models of COVID-19 Explain Inequities and Inform Reopening." Nature 589:82-87. https ://doi.org/10.1038/s41586-020-2923-3.

Chetty, Raj, John N. Friedman, Nathaniel Hendren, Michael Stepner, and The Opportunity Insights Team. 2020. "The Economic Impacts of COVID-19: Evidence from a New Public Database Built Using Private Sector Data." Technical Report 27431, National Bureau of Economic Research. 
Chetty, Raj, Nathaniel Hendren, and Lawrence F. Katz. 2016. "The Effects of Exposure to Better Neighborhoods on Children: New Evidence from the Moving to Opportunity Experiment." American Economic Review 106(4):855-902. https://doi .org/10.1257/aer . 20150572.

Coston, Amanda, Neel Guha, Derek Ouyang, Lisa Lu, Alexandra Chouldechova, and Daniel E. Ho. 2021. "Leveraging Administrative Data for Bias Audits: Assessing Disparate Coverage with Mobility Data for COVID-19 Policy." In Proceedings of the 2021 ACM Conference on Fairness, Accountability, and Transparency, FAccT '21, pp. 173-84, New York. Association for Computing Machinery.

Elbers, Benjamin. 2021. "A Method for Studying Differences in Segregation across Time and Space." Sociological Methods E Research, first published February 3, 2021. https: //doi.org/10.1177\%2F0049124121986204.

Feehan, Dennis M. and Ayesha S. Mahmud. 2021. "Quantifying Population Contact Patterns in the United States during the COVID-19 Pandemic." Nature Communications 12:893. https://doi.org/10.1038/s41467-021-20990-2.

Gao, Song, Jinmeng Rao, Yuhao Kang, Yunlei Liang, and Jake Kruse. 2020. "Mapping County-Level Mobility Pattern Changes in the United States in Response to COVID-19." SIGSPATIAL Special 12(1):16-26. https: //doi .org/10.1145/3404820.3404824.

Glaeser, Edward L., Caitlin Gorback, and Stephen J. Redding. 2020. “JUE Insight: How Much Does COVID-19 Increase with Mobility? Evidence from New York and Four Other U.S. Cities." Journal of Urban Economics, first published October 21, 2020. https ://doi .org/ $10.1016 / j$.jue.2020.103292.

Gupta, Sumedha, Thuy Nguyen, Felipe Lozano Rojas, Shyam Raman, Byungkyu Lee, Ana Bento, Kosali Simon, and Coady Wing. 2020. "Tracking Public and Private Responses to the COVID-19 Epidemic: Evidence from State and Local Government Actions." Technical Report 27027, National Bureau of Economic Research.

He, Jun, Leshui He, Wen Zhou, Xuanhua Nie, and Ming He. 2020. “Discrimination and Social Exclusion in the Outbreak of COVID-19." International Journal of Environmental Research and Public Health 17(8):2933. https ://doi .org/10.3390/ijerph17082933.

Kim, Junghwan, and Mei-Po Kwan. 2021. "How Neighborhood Effect Averaging Might Affect Assessment of Individual Exposures to Air Pollution: A Study of Ozone Exposures in Los Angeles." Annals of the American Association of Geographers 111(1):121-40. https: //doi.org/10.1080/24694452.2020.1756208.

Krivo, Lauren J., Heather M. Washington, Ruth D. Peterson, Christopher R. Browning, Catherine A. Calder, and Mei-Po Kwan. 2013. "Social Isolation of Disadvantage and Advantage: The Reproduction of Inequality in Urban Space." Social Forces 92(1):141-64. https://doi.org/10.1093/sf/sot043.

Krysan, Maria, and Kyle Crowder. 2017. Cycle of Segregation: Social Processes and Residential Stratification. New York: Russell Sage Foundation.

Levy, Brian L., Nolan E. Phillips, and Robert J. Sampson. 2020. “Triple Disadvantage: Neighborhood Networks of Everyday Urban Mobility and Violence in U.S. Cities." American Sociological Review 85(6):925-56. https://doi .org/10.1177\%2F0003122420972323. 
Liu, Luyu, Harvey J. Miller, and Jonathan Scheff. 2020. "The Impacts of COVID-19 Pandemic on Public Transit Demand in the United States." PLoS One 15(11):e0242476. https: //doi.org/10.1371/journal.pone.0242476.

Ma, Jing, Yinhua Tao, Mei-Po Kwan, and Yanwei Chai. 2020. "Assessing Mobility-Based Real-Time Air Pollution Exposure in Space and Time Using Smart Sensors and GPS Trajectories in Beijing." Annals of the American Association of Geographers 110(2):434-48. https://doi.org/10.1080/24694452.2019.1653752.

New York Times. 2021. "Coronavirus (Covid-19) Data in the United States." GitHub. https://github.com/nytimes/covid-19-data.

Newport, Frank. 2020. "Race Relations as the Nation's Most Important Problem." Gallup. https://news.gallup.com/opinion/polling-matters/312875/racerelations-nation-important-problem.aspx.

Nguyen, Thuy D., Sumedha Gupta, Martin Andersen, Ana Bento, Kosali I. Simon, and Coady Wing. 2020. "Impacts of State Reopening Policy on Human Mobility." Technical Report 27235, National Bureau of Economic Research.

Nouvellet, Pierre, Sangeeta Bhatia, Anne Cori, Kylie E. C. Ainslie, Marc Baguelin, Samir Bhatt, Adhiratha Boonyasiri, Nicholas F. Brazeau, Lorenzo Cattarino, Laura V. Cooper, Helen Coupland, Zulma M. Cucunuba, Gina Cuomo-Dannenburg, Amy Dighe, Bimandra A. Djaafara, Ilaria Dorigatti, Oliver D. Eales, Sabine L. van Elsland, Fabricia F. Nascimento, Richard G. FitzJohn, Katy A. M. Gaythorpe, Lily Geidelberg, William D. Green, Arran Hamlet, Katharina Hauck, Wes Hinsley, Natsuko Imai, Benjamin Jeffrey, Edward Knock, Daniel J. Laydon, John A. Lees, Tara Mangal, Thomas A. Mellan, Gemma Nedjati-Gilani, Kris V. Parag, Margarita Pons-Salort, Manon Ragonnet-Cronin, Steven Riley, H. Juliette T. Unwin, Robert Verity, Michaela A. C. Vollmer, Erik Volz, Patrick G. T. Walker, Caroline E. Walters, Haowei Wang, Oliver J. Watson, Charles Whittaker, Lilith K. Whittles, Xiaoyue Xi, Neil M. Ferguson, and Christl A. Donnelly. 2021. "Reduction in Mobility and COVID-19 Transmission." Nature Communications 12:1090. https://doi .org/10.1038/s41467-02121358-2.

Papachristos, Andrew V., Chris M. Smith, Mary L. Scherer, and Melissa A. Fugiero. 2011. "More Coffee, Less Crime? The Relationship between Gentrification and Neighborhood Crime Rates in Chicago, 1991 to 2005." City \& Community 10(3):215-40. https : //doi .org/ 10.1111\%2Fj. 1540-6040.2011.01371.x.

Perchoux, Camille, Basile Chaix, Steven Cummins, and Yan Kestens. 2013. “Conceptualization and Measurement of Environmental Exposure in Epidemiology: Accounting for Activity Space Related to Daily Mobility." Health E Place 21:86-93. https: //doi.org/10.1016/j.healthplace.2013.01.005.

Pettigrew, Thomas F. 1998. "Intergroup Contact Theory." Annual Review of Psychology 49:65-85. https://doi.org/10.1146/annurev.psych.49.1.65.

Pew Research Center. 2021. "Demographics of Mobile Device Ownership and Adoption in the United States." Pew Research Center. https ://www .pewresearch.org/internet/ fact-sheet/mobile/.

Phillips, Nolan E., Brian L. Levy, Robert J. Sampson, Mario L. Small, and Ryan Q. Wang. 2019. "The Social Integration of American Cities: Network Measures of Connectedness Based on Everyday Mobility across Neighborhoods." Sociological Methods E Research. first published July 17, 2019. https : //doi .org/10.1177\%2F0049124119852386. 
Prestby, Timothy, Joseph App, Yuhao Kang, and Song Gao. 2020. "Understanding Neighborhood Isolation through Spatial Interaction Network Analysis Using Location Big Data." Environment and Planning A: Economy and Space 52(6):1027-31. https ://doi .org/10.1177\% 2F0308518X19891911.

Reny, Tyler T., and Matt A. Barreto. 2020. "Xenophobia in the Time of Pandemic: Othering, Anti-Asian Attitudes, and COVID-19." Politics, Groups, and Identities, first published May 28, 2020. https://doi.org/10.1080/21565503.2020.1769693.

Rizzuto, Max. 2020. “U.S. Politicians Exploit Coronavirus Fears with Anti-Chinese Dog Whistles." Digital Forensic Research Lab. https://www.atlanticcouncil.org/ commentary/article/u-s-politicians-exploit-coronavirus-fears-with-antichinese-dog-whistles/.

SafeGraph. 2021a. "Places Manual." https://docs.safegraph.com/v4.0/docs/placesmanual.

SafeGraph. 2021b. "Social Distancing Metrics." https://docs.safegraph.com/docs/ social-distancing-metrics.

Shamshiripour, Ali, Ehsan Rahimi, Ramin Shabanpour, and Abolfazl (Kouros) Mohammadian. 2020. "How Is COVID-19 Reshaping Activity-Travel Behavior? Evidence from a Comprehensive Survey in Chicago." Transportation Research Interdisciplinary Perspectives 7:100216. https://doi.org/10.1016/j.trip.2020.100216.

Sharkey, Patrick, and Jacob W. Faber. 2014. “Where, When, Why, and for Whom Do Residential Contexts Matter? Moving Away from the Dichotomous Understanding of Neighborhood Effects." Annual Review of Sociology 40:559-79. https : //doi . org/10 .1146/annurevsoc-071913-043350.

Sharp, Gregory, Justin T. Denney, and Rachel T. Kimbro. 2015. “Multiple Contexts of Exposure: Activity Spaces, Residential Neighborhoods, and Self-Rated Health." Social Science $\mathcal{E}$ Medicine 146:204-13. https://doi.org/10.1016/j.socscimed.2015.10.040.

Squire, Ryan Fox. 2019. "What about Bias in the SafeGraph Dataset?" SafeGraph. https : //www.safegraph.com/blog/what-about-bias-in-the-safegraph-dataset.

Sugie, Naomi F., and Michael C. Lens. 2017. "Daytime Locations in Spatial Mismatch: Job Accessibility and Employment at Reentry from Prison." Demography 54:775-800. https://doi.org/10.1007/s13524-017-0549-3.

Tavernise, Sabrina, and Richard A. Oppel, Jr. 2020. "Spit On, Yelled At, Attacked: ChineseAmericans Fear for Their Safety." New York Times, March 23, 2020. https : //www .nytimes . com/2020/03/23/us/chinese-coronavirus-racist-attacks.html.

Wang, Qi, Nolan Edward Phillips, Mario L. Small, and Robert J. Sampson. 2018. "Urban Mobility and Neighborhood Isolation in America's 50 Largest Cities." Proceedings of the National Academy of Sciences 115(3):7735-40. https : / doi .org/10.1073/pnas . 1802537115.

Weeden, Kim A., and Ben Cornwell. 2020. "The Small-World Network of College Classes: Implications for Epidemic Spread on a University Campus." Sociological Science 7:222-41. https://doi.org/10.15195/v7.a9. 
Acknowledgments: We would like to thank Byungkyu Lee, Philipp Brandt, and Clara G. Sears for their insightful feedback on earlier drafts. This work was supported by the NYUAD Center for Interacting Urban Networks (CITIES), funded by Tamkeen under the NYUAD Research Institute Award CG001 and by the Swiss Re Institute under the Quantum Cities initiative. Bruno Abrahao was supported by National Natural Science Foundation of China (NSFC) Grant 61850410536.

Thomas Marlow: Center for Interacting Urban Networks (CITIES), New York University Abu Dhabi. E-mail: twm9710@nyu.edu.

Kinga Makovi: Social Science Division, New York University Abu Dhabi.

E-mail: km2537@nyu.edu.

Bruno Abrahao: Business Division, New York University Shanghai; Global Network Assistant Professor, Leonard N. Stern School of Business, New York University. E-mail: bd58@nyu.edu. 